\title{
Approximating Optimal Release in a Deterministic Model for the Sterile Insect Technique
}

\author{
Sergio Ramirez and Luis F. Gordillo \\ Department of Mathematics and Statistics, Utah State University, Logan, UT 84322, USA \\ Correspondence should be addressed to Luis F. Gordillo; luis.gordillo@usu.edu
}

Received 24 November 2015; Revised 12 February 2016; Accepted 14 February 2016

Academic Editor: Rodomiro Ortiz

Copyright (C) 2016 S. Ramirez and L. F. Gordillo. This is an open access article distributed under the Creative Commons Attribution License, which permits unrestricted use, distribution, and reproduction in any medium, provided the original work is properly cited.

\begin{abstract}
Cost/benefit analyses are essential to support management planning and decisions before launching any pest control program. In particular, applications of the sterile insect technique (SIT) are often prevented by the projected economic burden associated with rearing processes. This has had a deep impact on the technique development and its use on insects with long larval periods, as often seen in beetles. Under the assumptions of long adult timespan and multiple mating, we show how to find approximate optimal sterile release policies that minimize costs. The theoretical framework proposed considers the release of insects by pulses and finds approximate optimal release sizes through stochastic searching. The scheme is then used to compare simulated release strategies obtained for different pulse schedules and release bounds, providing a platform for evaluating the convenience of increasing sterile male release intensity or extending the period of control.
\end{abstract}

\section{Introduction}

The use of ionizing radiation, genetic modification, or exposure to chemicals to induce sterility in male insects, which are later released in the wild, is considered an ideal pest control method because of its harmless impact on the environment and absence of detrimental consequences for human health [1]. In particular, the effects of irradiation have been extensively studied for agricultural purposes on hundreds of arthropod species in an attempt to identify cases where the technique can be conveniently applied [2]. Although the release of sterile insects has been successful in controlling pest outbreaks in many cases [1], for most pest species issues related to mass-rearing and biological constraints often affect the feasibility of the technique [1-3]. For instance, rearing coleopteran insects with relatively long larval periods is exceedingly expensive, and males of some lepidopteran species face an important reduction in their ability to mate after irradiation.

In pest control programs, one of the main decisionmaking challenges is to determine economically feasible levels of mass-rearing that may drive pest populations to extinction. This search often uses deterministic, stochastic, or computational models that have been successfully developed during the last decades to predict or explain the behavior of insect populations in presence of sterile males [4-7]. Minimum effective sterile production is sometimes easily approximated if the theoretical model used is simple enough [4]; however, the model complexity might increase dramatically when specific biological details are to be incorporated or when production constraints are to be taken into account.

This paper provides a conceptual framework for searching the optimal rearing levels necessary for eliminating a pest outbreak for the particular case of insects with relatively long lifespan and multiple matings, as observed frequently in species of beetles. The motivation comes from promising results that have been obtained in pilot control programs tested against cockchafers (Melolontha vulgaris) [8], the boll weevil (Anthonomus grandis) [9-11], and sweet potato weevils (Cylas formicarius) [12]. Remarkably in the latter case, carried out at Kumo Island, Okinawa Prefecture in Japan, the eradication with SIT was complete. Following that success, SIT is being tested on Euscepes postfasciatus, another sweet potato weevil pest in the island. Nevertheless, as mentioned before, a primary drawback for generalized application of SIT has been long larval periods that make rearing costs high.

Our approximations to optimal sterile male release are made using a continuous time population model developed 
in [13]. The differential equations considered capture the emergence of a reproductive Allee effect that is enhanced by the release of sterile males: mate finding becomes more difficult for wild males due to competition with introduced sterile males and females' overall capacity to produce offspring is reduced $[14,15]$. Thus we notice that the use of emerging Allee effects as part of population control practices falls into the scope of integrated pest management. The conceptual model introduced here allows computing sterile release policies aimed at reducing the pest population to a level at which Allee effects naturally take off and conclude the eradication. We remark that sterile release is integrated into the model via pulses that follow a predetermined schedule. Finally, we explore numerically the effects on optimal sterile release produced by (i) variations in the pulse scheduling and (ii) changes in the release bounds of sterile males. By comparing the results from the computations it might be possible to determine suitable release strategies for control program planning.

\section{Model Framework}

We use a continuous time model developed in [13] for the sexually mature female pest population. It specifically addresses the situation where the average lifespan is considerably larger than the average time spent at the reproductive stage, understood here as the immediate postcopulatory stage at which females are not sexually receptive. This is in agreement with the sharp loss of receptiveness that appears generally as a physiological response to male materials passed during copulation [3]. Biologically, this fact assures the possibility of multiple mating encounters and plays an important role in the model reduction.

First we establish the following biological reasonable assumptions: (i) different sexes are homogeneously mixed, (ii) irradiation is not detrimental to the mating ability in sterile males, (iii) females mate at random and the total average number of successful mating encounters is proportional to the number of males (sterile and wild), (iv) the sex ratio is constant $1: 1$, and (v) sterile insects mix homogeneously within the whole population immediately after they are released. We also suppose that density regulation mechanisms have negligible impact on the population, an assumption that would be in agreement with the initial stages of many invasion process.

We consider a particular group of adult females, $N$, composed of those individuals that mated successfully at least once with a wild male. The density of this group at time $t$ is denoted here with $N(t)$. Females in this group are further classified in three categories:

(i) $N_{1}$ : females at reproductive stage, impregnated by a wild male,

(ii) $\mathrm{N}_{2}$ : females ready for mating,

(iii) $N_{3}$ : females at reproductive stage, impregnated by a sterile male.

Females in category $N_{2}$ will change their status and move to either $N_{1}$ or $N_{3}$, depending on if they mate with a wild or

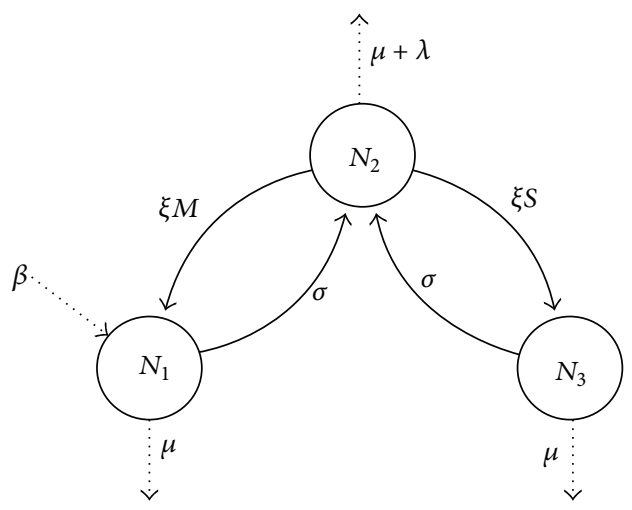

FIGURE 1: Schematic dynamics between female categories $N_{1}$ (reproductive stage after mating with a wild male), $N_{2}$ (ready to mate), and $N_{3}$ (reproductive stage after mating with a sterile male). $M$ and $S$ represent the density of wild and sterile males, respectively, $\mu$ is the death rate, $1 / \beta$ is the average time a young female waits until its first mating encounter with a wild male, $1 / \sigma$ is the average time that a female spends gravid, $\lambda$ is an additional mortality term, and $\xi$ represents the per capita average rate of mating encounters.

sterile male, respectively. They will naturally be ready to mate again after some time; that is, they will return to compartment $N_{2}$. Let $M$ be the density of adult wild males that are sexually mature and $S$ the density of sterile males. We then choose to model the flow of females between classes $N_{1}, N_{2}$, and $N_{3}$ by the following system of equations:

$$
\begin{aligned}
& N_{1}^{\prime}=(\beta-\mu) N_{1}-\sigma N_{1}+\xi M N_{2}, \\
& N_{2}^{\prime}=-(\mu+\lambda) N_{2}+\sigma\left(N_{1}+N_{3}\right)-\xi(M+S) N_{2}, \\
& N_{3}^{\prime}=-\mu N_{3}-\sigma N_{3}+\xi S N_{2},
\end{aligned}
$$

where $\mu$ represents the death rate; $1 / \beta$ is the average time a young female adult waits until its first mating encounter with a wild male occurs; $1 / \sigma$ is the average time that a female spends in the reproductive stage; that is, $\sigma$ is the rate of return to mating status; and $\lambda$ is an additional mortality term due, for instance, to an increased exposure to predators while searching for a mate. The rate at which females turn gravid through mating with wild or sterile males is given by $\xi(M+S) N_{2}$, where $\xi$ represents the per capita average rate of mating encounters. See Figure 1 for schematic representation of female flow between categories.

Remark 1. By allowing new adult females to get into the group $N$ at the moment they have their first successful mating contact with a wild male we exclude those females that will only mate with sterile males and do not contribute to population growth. It might also be possible to let young adult females to get into $N$ through the category $N_{2}$ instead but the differences between the solutions to both models are negligible and only present in an initial transient.

Recalling that the sex ratio is assumed to be even and under the assumption that the average adult timespan is much larger than the average time at the reproductive stage it 
is possible to approximate the system of differential equations (1) by a single equation involving only the variable $N$; see [13] for mathematical details:

$$
N^{\prime}=\phi N\left(1-\frac{\eta(1+\theta S)}{1+\gamma(N+S)}\right),
$$

with

$$
\begin{aligned}
& \phi=\beta-\mu, \\
& \eta=\frac{\sigma(\beta+\lambda)}{(\beta-\mu)(\sigma+\lambda)}, \\
& \gamma=\frac{\xi}{\sigma+\lambda}, \\
& \theta=\frac{\beta \xi}{\sigma(\beta+\lambda)} .
\end{aligned}
$$

Equation (2) has an unstable positive equilibrium in addition to the origin. It marks a threshold under which the population would drop due to a reproductive Allee effect that has been enhanced by sterile male release. We remark that the value of this threshold depends on the contact rate $\xi$ [13].

We assume now that sterile males are released by pulses, in contrast with the models in [13], where the population of sterile insects is kept constant through a continuous influx that compensates their death. Let us call the time between sterile release pulses the interpulse time, which we assume to be fixed and equal to $T$. During these time intervals, the sterile male population will decrease at a certain rate, $\mu_{S}$, not necessarily equal to that of wild males. Thus, the release of sterile males can be modeled using a set of impulsive equations [16], where the $n$th pulse adds to the sterile population density $X_{n} \geq 0$ individuals:

$$
S^{\prime}=-\mu_{S} S \quad \text { if } n T \leq t<(n+1) T, n=0,1,2, \ldots,
$$

with an initial value $S_{0}=S(0)$ and

$$
S((n+1) T)=S\left(n T^{-}\right)+X_{n}, \quad n=1,2, \ldots
$$

It is apparent that the pest regulation dynamics should rest upon the control over the amount of sterile insects released and the interpulse time, that is, $X_{n}$ 's and $T$. Let us assume in general that the amount of sterile males released is the same amount produced. Then, for the pulses, we introduce a constrained production $0 \leq X_{n} \leq B$ due, for instance, to economic limitations. Notice, however, that the bound $B$ might not be the same for each pulse and that the design of a control program might require eradication or suppression within a given time frame.

Among the solutions of (2), (4), and (5) for which the final female population is less than a desired fixed level, we now look for the one that requires the minimum release of sterile males. If there are $m$ scheduled pulses, let us set $\mathbf{X}=$ $\left(X_{0}, \ldots, X_{m-1}\right)$; then we have the problem of finding

$$
\min _{\mathbf{X}} \sum_{0}^{m-1} X_{n}
$$

subject to a predetermined final female population, which might be the insect detectable value if the goal is eradication. Notice that losses due to pest damage are not considered in (6). This can be amended by the use of a more general cost function, depending continuously on time, established as follows. Let $C(t)$ denote the total costs at time $t$, understood here as having two components: the cost of producing sterile males and the losses due to the damage inflicted by the pest. We begin assuming, as before, that the change in cost per unit of time, $C^{\prime}(t)$, is directly proportional to the amount of sterile males produced per unit of time, plus the money lost in damaged crops to the insects at a certain rate:

$$
C^{\prime}(t)=P \widehat{X}(t)+F N(t)
$$

where $P$ is the cost per sterile male, $\widehat{X}(t)$ is the rate of production of sterile males, and $F$ is the rate at which money is lost to feeding per pest. This equation governs the rate at which the cost is changing over time. Since both $\widehat{X}(t)$ and $N(t)$ are positive, $C^{\prime}(t)$ is always positive and $C(t)$ is nondecreasing. The total cost up to a time $t$ can be expressed as

$$
C(t)=\int_{0}^{t} P \widehat{X}(\tau)+F N(\tau) d \tau
$$

which becomes

$$
C(t)=\sum_{0}^{m-1} P X_{n}+\int_{0}^{t} F N(\tau) d \tau,
$$

within the context of pulse release, up to time $t \geq m T$. Given that we control the rate at which sterile males are being released, we want to find a suitable balance in which we do not spend too much money in the production but also do not lose most of the crop to feeding. Therefore, the problem is to minimize $C(t)$ over all permissible controls $\mathbf{X}=\left(X_{0}, \ldots, X_{m-1}\right)$, subject to (i) the predetermined final female population and (ii) the differential equations

$$
N^{\prime}(\tau)=\phi N\left(1-\frac{\eta\left(1+\theta Q_{n} e^{-\mu_{S} \tau}\right)}{1+\gamma\left(N+Q_{n} e^{-\mu_{S} \tau}\right)}\right)
$$

on $[n T,(n+1) T), n=0,1,2, \ldots$, where $Q_{n}=S\left(n T^{-}\right)+X_{n}=$ $Q_{n-1} e^{-\mu_{S} T}+X_{n}, n=1,2, \ldots$, with $Q_{0}=S(0)+X_{0}$. Constraint (ii) is a "chain" of differential equations, at each given time period we have a differential equation with initial conditions determined by the final values of the variable $N$ and the corresponding $Q$ from the previous period.

\section{Optimization by Simulated Annealing}

The natural approach to these kinds of optimal control problems is to try Pontryagin's principle [17]. Unfortunately in this case it leads to cumbersome analytical expressions. Instead, we opt to solve the optimization problem using a particular instance of stochastic search, simulated annealing (SA), for which we give here a brief description; see [18] for a detailed account. In general, the SA algorithm can be used 

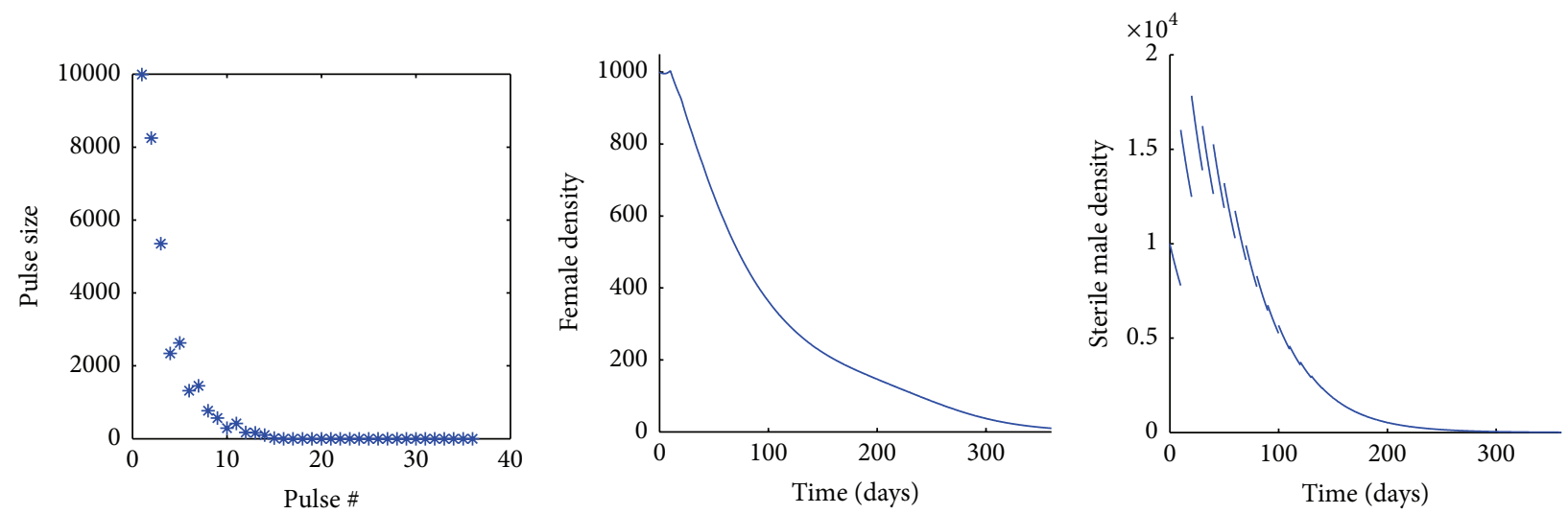

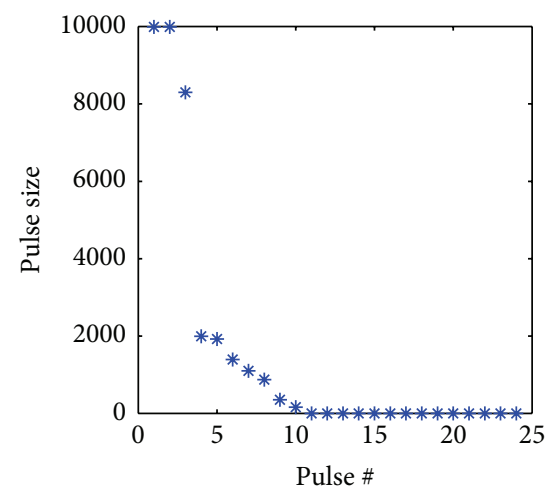

(a)

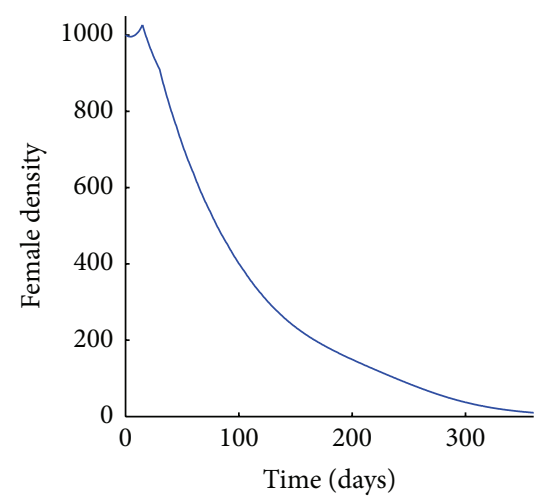

(b)

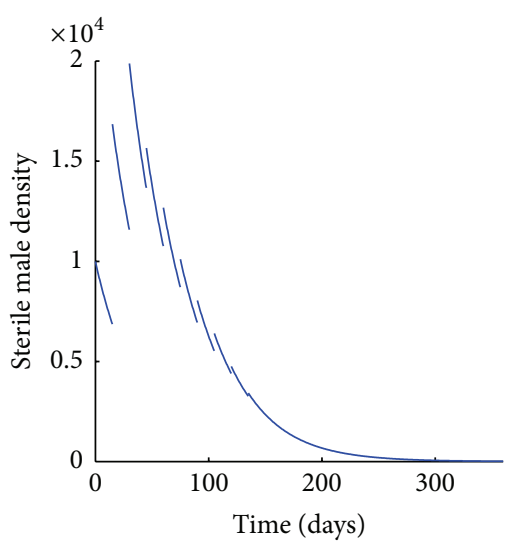

(c)

FIGURE 2: (a) Approximations to optimal sterile male pulse sizes obtained assuming that the maximum release of insects is limited to ten times the initial density (taken equal to 1000). ((b) and (c)) Progress of female and sterile male densities, with pulses of sterile males made every ten days (top) and fifteen days (bottom). Notice that (i) the maximum release is required only at the beginning stages, until the sterile population overflows the wild population and (ii) release of sterile males is not required after the Allee effect takes place and drives the remaining pest population to extinction.

to solve the optimization problem $\min f(x)$ over all $x \in \mathscr{C}$, where $f$ is a real valued function and $\mathscr{C}$ is a set of feasible states (controls). We apply the SA algorithm having as the set of feasible states finite dimensional arrays with subsequent sterile production levels as components and introduce an arbitrary initial control $\mathbf{X}_{0}$. Using this, first we solve the differential equations numerically for $N(t)$. Once we have obtained $N(t)$, we use these values along with the guessed $\mathbf{X}_{0}$ to evaluate the objective functional and obtain the cost, denoted by $C_{0}$. Then we repeat all the previous steps, but this time we use a different feasible control $\mathbf{X}$ to obtain a different cost $C$ and compare the two cost function values. If $C<C_{0}$, replace $C_{0}$ with $C$. If, on the other hand, $C>C_{0}$, we do not automatically throw $C$ away. Instead we calculate $p=\exp \left(-\left(C-C_{0}\right) / \tau\right)$, where $\tau$ is a "temperature" parameter. If $p>U$, where $U$ is a uniform random generated number between zero and one, replace $C_{0}$ with $C$.

\section{Example}

With the population dynamics equations and the optimization technique at hand, we are in position of addressing for specific cases two questions: (i) what is the minimum sterile release necessary at each pulse that would induce pest eradication at the end of a given time window? (ii) How does the strategy change in the face of variations on the interpulse time or release bounds?

To illustrate how to proceed, we first set the realistic parameter values $1 / \mu=40$ (day), $\beta=0.3\left(\right.$ day $\left.^{-1}\right)$ and average reproductive time of $1 / \sigma=1 / 2$ (day), which are consistent with averages found for beetles [13]. For sterile males we assume $\mu_{S}=\mu$ although, as already mentioned, this is not necessarily true because the value of $\mu_{S}$ depends on the particular species response to irradiation doses. In addition, we arbitrarily choose $\lambda=0$ and $\xi=0.001\left(\right.$ day $\left.^{-1}\right)$.

Effects of Changing Interpulse Time. We arbitrarily assume a bound on the sterile male release equal to ten times the initial amount of wild females, which is taken equal to 1000 . We recall at this point that we have also assumed the numbers of sterile males produced and released as equal. Then we compute approximations to the optimal release for two cases: when pulses of sterile males are scheduled every ten and fifteen days for a complete year. At the end of this time window, the wild female population is required to be less than the insect detectable value. In our computations, for 

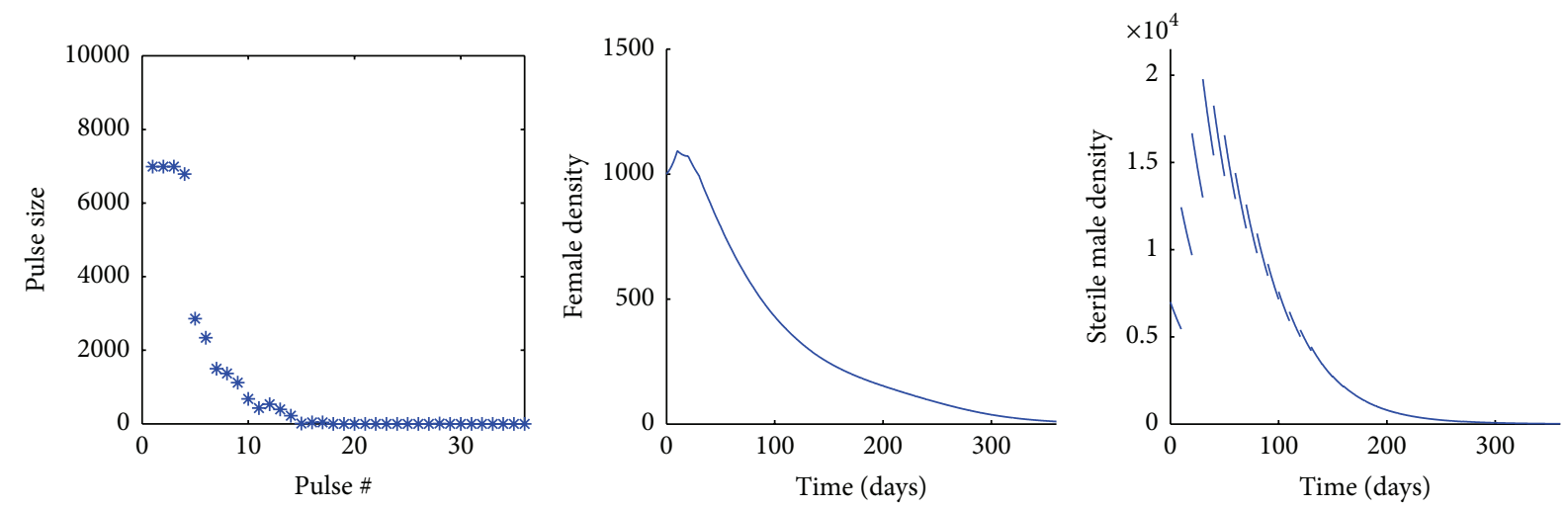

(a)
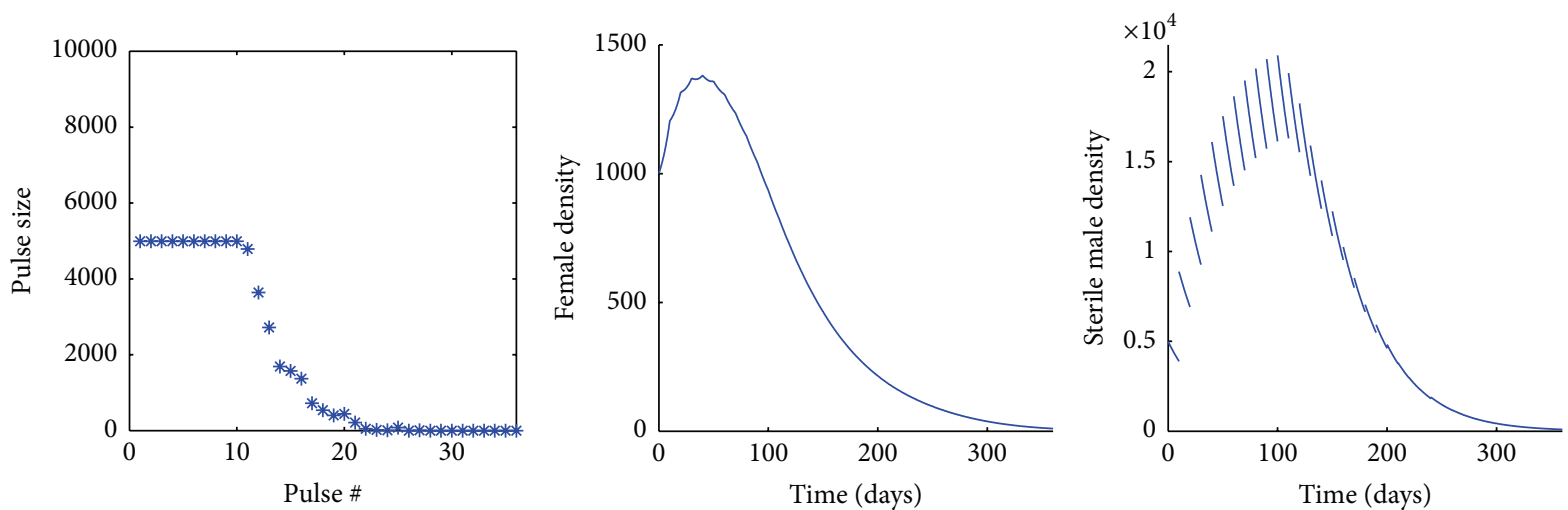

(b)

Figure 3: With interpulse time fixed to 10 days we now vary sterile release bounds to 7000 (a) and 5000 (b). Intense and prolonged sterile release is required during the first stages until the pest growth tendency is reversed. Notice that the Allee threshold takes longer to appear. Changing the problem with an interpulse time of 15 days did not produce a solution; that is, invasion is inevitable.

example, we required the final female density to be less than ten. The numerical results are displayed in Figure 2. After an initial transient where the releases for the first pulses have to be relatively high, pulse sizes are seen to decrease until the female density reaches an Allee threshold that drives the population into a natural decline. In other words, sterile male release is not necessary for the whole year due to the pressure of remanent sterile males in combination with the low female density. One difference however is that the release of high quantities of sterile males for the second case, that is, when the interpulse time is longer, has to be prolonged during the initial stages. The interpretation is that the sterile density is growing slower so females have, at least initially, larger probability of mating with wild males. This implies that the tendency to increase the pest population growth has to be more intense at the beginning. The costs incurred by the production of sterile males with interpulse time $T, C_{T}$, can also be computed. For the example it is determined that $C_{15}>C_{10}$, as it would be expected.

Effects of Changing Bounds for Sterile Release. Now let us fix the interpulse time in ten days and change the maximum amount of sterile release at each pulse. As illustration, we choose arbitrarily bounds of 7000 and 5000 sterile males per pulse. The results are shown in Figure 3. We notice that the time to reach the necessary sterile density to revert the pest tendency to growth is longer when the bound is lower; that is, a longer and intense release policy at the initial stages is needed. Also, the Allee threshold takes longer to be reached. It was not possible to find a solution to the problem that uses the second bound and extend the interpulse time to 15 days, suggesting that the time window of control application has to be extended.

\section{Conclusions}

The management of insect pests in agriculture is primarily done through the use of insecticides, biological control, or crop rotation. It is natural for these methods to be modeled through impulsive differential equations due to their relatively large and instantaneous effects; see, for instance, [19$22]$. This paper is concerned with the pulse release of sterile males for an insect population where multiple matings take place and females have relatively short copulatory stages. We have shown how the minimum size of pulses that suppress a given insect population could be approximated and how these results are affected under variation in interpulse time and release bounds. The conceptual framework used involves a novel population model that addresses the specific biological 
characteristics together with the application of stochastic search.

Unfortunately in practice biological constraints in the rearing process, like long larval stages, might contribute to making the sterile insect release economically disadvantageous. Therefore, there is usefulness of having prior information on the optimal sterile pulses that induce extinction. Computing the optimal magnitude of the sterile release pulses for different interpulse times allows comparing strategies and ultimately determining the best path to follow for a suitable control planning.

We illustrate the use of our scheme with an example that has biologically sound parameters: the outputs obtained from different interpulse time regimes and different release bounds are contrasted. The results show (i) how the required intensity of the sterile release depends on interpulse times and (ii) the appearance of a delay in the Allee effect due to variations on the sterile release bounds. In general, the scheme can be used in species-specific cases to determine if a constrained sterile release is suitable for reducing the pest population and how long will it take to do that in relation to interpulse times. Cost functions that include quantification of the economic damage by the pest can be implemented easily in the algorithm.

The approach in this paper does not seem to have been used before in pest control contexts, where difficult similar optimization problems abound. We emphasize, however, that deep knowledge about the target pest species biology is needed before any application of the scheme proposed. This information is crucial if we want to find whether the use of SIT in an area-wide integrated pest management program is suitable [3]. The conceptual model presented might serve well as a qualitative guide to forecast pest dynamics rather than an exact quantitative tool. Further numerical explorations integrating density dependence and spatial spread of insects [13] and the sensitivity of the results to the model parameters could be addressed. Although our main focus is on species with short copulatory stages and relatively long adult timespan, the possibility of applying the optimal search to species with short lifespan using model (1) is not precluded. Finally, we remark that the scheme proposed also provides a venue to study the impact of managerial decisions under the combined use of SIT with other control measures, like the use of pheromone traps.

\section{Conflict of Interests}

The authors declare that there is no conflict of interests regarding the publication of this paper.

\section{References}

[1] W. Klassen and C. F. Curtis, "History of the sterile insect technique," in Sterile Insect Technique. Principles and Practice in Area-Wide Integrated Pest Management, V. A. Dyck, J. Hendrichs, and A. S. Robinson, Eds., pp. 3-36, Springer, Amsterdam, Netherlands, 2005.

[2] A. Bakri, K. Mehta, and D. R. Lance, "Sterilizing insects with ionizing radiation," in Sterile Insect Technique. Principles and Practice in Area-Wide Integrated Pest Management, V. A. Dyck,
J. Hendrichs, and A. S. Robinson, Eds., pp. 233-258, Springer, Amsterdam, Netherlands, 2005.

[3] D. R. Lance and D. O. McInnis, "Biological basis of the sterile insect technique," in Sterile Insect Technique: Principles and Practice in Area-Wide Integrated Pest Management, V. A. Dyck, J. Hendrichs, and A. S. Robinson, Eds., pp. 69-94, Springer, Dordrecht, The Netherlands, 2005.

[4] H. J. Barclay, "Mathematical models for the use of sterile insects," in Sterile Insect Technique: Principles and Practice in Area-Wide Integrated Pest Management, V. A. Dyck, J. Hendrichs, and A. S. Robinson, Eds., pp. 147-174, Springer, Dordrecht, The Netherlands, 2005.

[5] H. Barclay and M. Mackauer, "The sterile insect release method for pest control: a density-dependent model," Environmental Entomology, vol. 9, no. 6, pp. 810-817, 1980.

[6] W. G. Costello and H. M. Taylor, "Mathematical models of the sterile male technique of insect control," in Mathematical Analysis of Decision Problems in Ecology, A. Charnes and W. R. Lynn, Eds., vol. 5 of Lecture Notes in Biomathematics, pp. 318359, Springer, Berlin, Germany, 1975.

[7] T. Yamanaka and A. M. Liebhold, "Spatially implicit approaches to understand the manipulation of mating success for insect invasion management," Population Ecology, vol. 51, no. 3, pp. 427-444, 2009.

[8] E. Horber, "Eradication of the white grub (Melolontha vulgaris F.) by the sterile male technique," in Proceedings of the Symposium: Radiation and Radioisotopes Applied to Insects of Agricultural Importance, STI/PUB/74, pp. 313-332, IAEA, Vienna, Austria, 1963.

[9] W. Klassen and N. W. Earle, "Permanent sterility induced in boll weevils with busulfan without reducing production of pheromone," Journal of Economic Entomology, vol. 63, no. 4, pp. 1195-1198, 1970.

[10] E. F. Knipling, The Basic Principles of Insect Population Suppression and Management, Agriculture Handbook Number 512, SEA, USDA, Washington, DC, USA, 1979.

[11] W. Klassen, Eradication of Introduced Arthropod Pests: Theory and Historical Practice, Miscellaneous Publications no. 73, Entomological Society of America, 1989.

[12] T. Kohama, M. Yamagishi, H. Kuba, and K. Kinjo, "A progress report on the eradication program of the sweet potato weevil, Cylas formicarius (Fabricius) (Coleoptera: Brentidae), with both male annihilation using sex pheromone and sterile insect releases in Kume Island, Okinawa, Japan," in Recent Trends on Sterile Insect Technique and Area-Wide Integrated Pest Management-Economic Feasibility, Control Projects, Farmer Organization and Bactrocera dorsalis Complex Control Study, pp. 65-69, Research Institute for Subtropics, Okinawa, Japan, 2003.

[13] L. F. Gordillo, "Pest persistence and eradication conditions in a deterministic model for sterile insect release," Journal of Biological Dynamics, vol. 9, supplement 1, pp. 64-78, 2015.

[14] F. Courchamp, L. Berec, and J. Gascoigne, Allee Effects in Ecology and Conservation, Oxford University Press, New York, NY, USA, 2008.

[15] L. F. Gordillo, "Modeling ephemeral mating encounters in insects: the emergence of mate-finding Allee effects and applications to theoretical models of sterile release," Theoretical Population Biology, vol. 104, pp. 10-16, 2015.

[16] L. Mailleret and V. Lemesle, "A note on semi-discrete modelling in the life sciences," Philosophical Transactions of the Royal Society of London A, vol. 367, no. 1908, pp. 4779-4799, 2009. 
[17] M. Athans and P. Falb, Optimal Control, Dover, Mineola, NY, USA, 2007.

[18] J. C. Spall, Introduction to Stochastic Search and Optimization: Estimation, Simulation, and Control, Wiley-Interscience Series in Discrete Mathematics, Wiley-Interscience, Hoboken, NJ, USA, 2003.

[19] V. A. A. Jansen and M. W. Sabelis, "Outbreaks of colony-forming pests in tri-trophic systems: consequences for pest control and the evolution of pesticide resistance," Oikos, vol. 74, no. 1, pp. 172-176, 1995.

[20] B. Liu, L. S. Chen, and Y. J. Zhang, "The dynamics of a preydependent consumption model concerning impulsive control strategy," Applied Mathematics and Computation, vol. 169, no. 1, pp. 305-320, 2005.

[21] Z. Lu, X. Chi, and L. Chen, "Impulsive control strategies in biological control of pesticide," Theoretical Population Biology, vol. 64, no. 1, pp. 39-47, 2003.

[22] R. Shi and L. Chen, "Staged-structured Lotka-Volterra predatorprey models for pest management," Applied Mathematics and Computation, vol. 203, no. 1, pp. 258-265, 2008. 


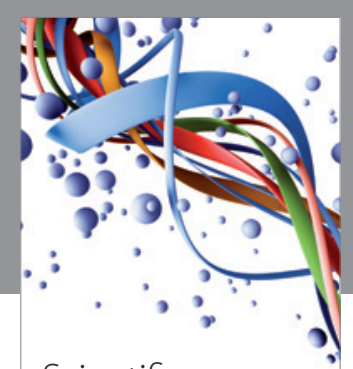

Scientifica
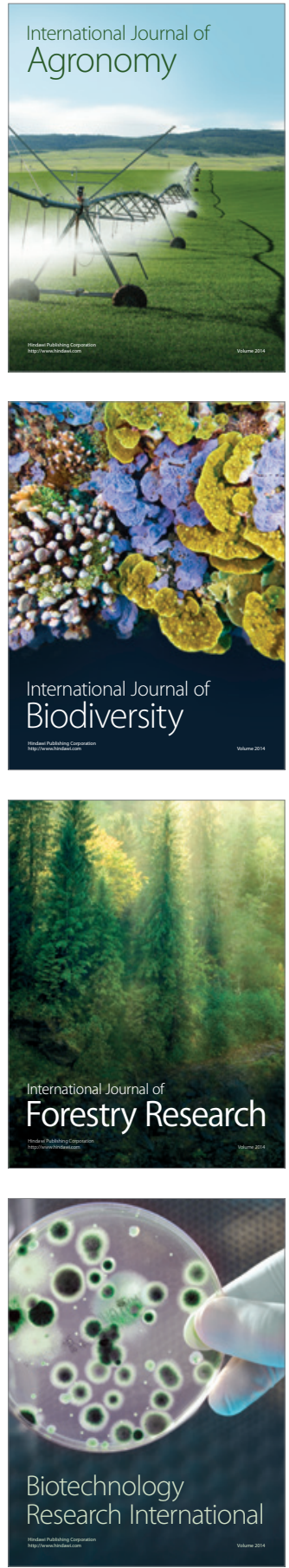
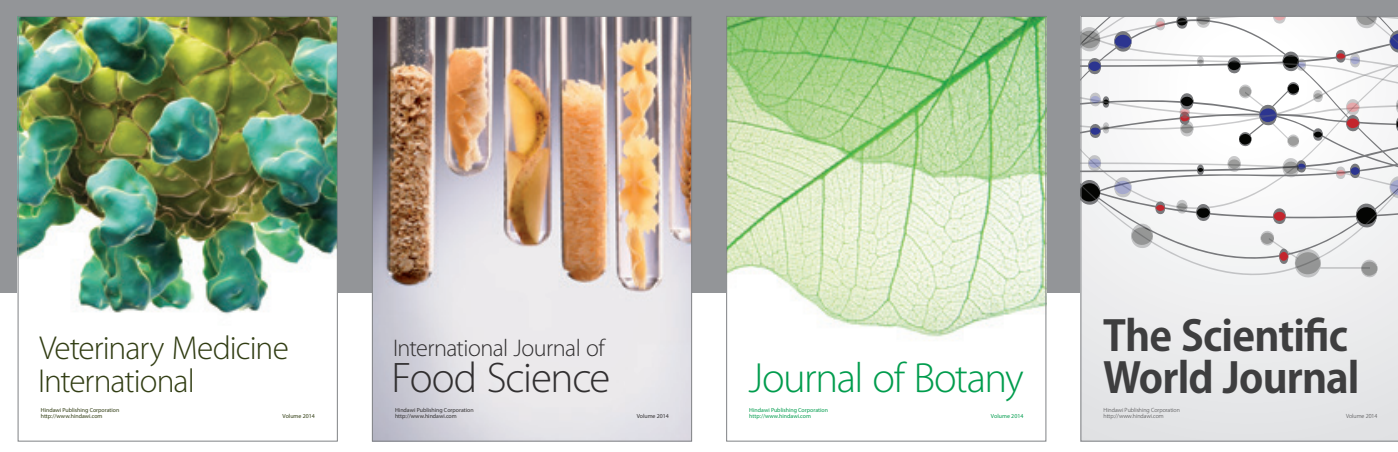

The Scientific

\section{World Journal}

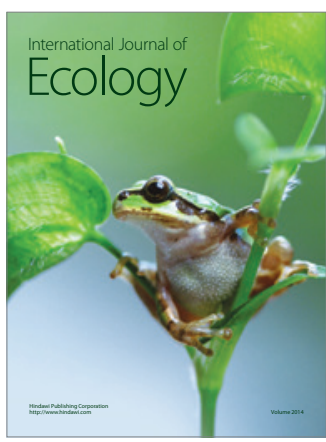

\section{Hindawi}

Submit your manuscripts at

http://www.hindawi.com
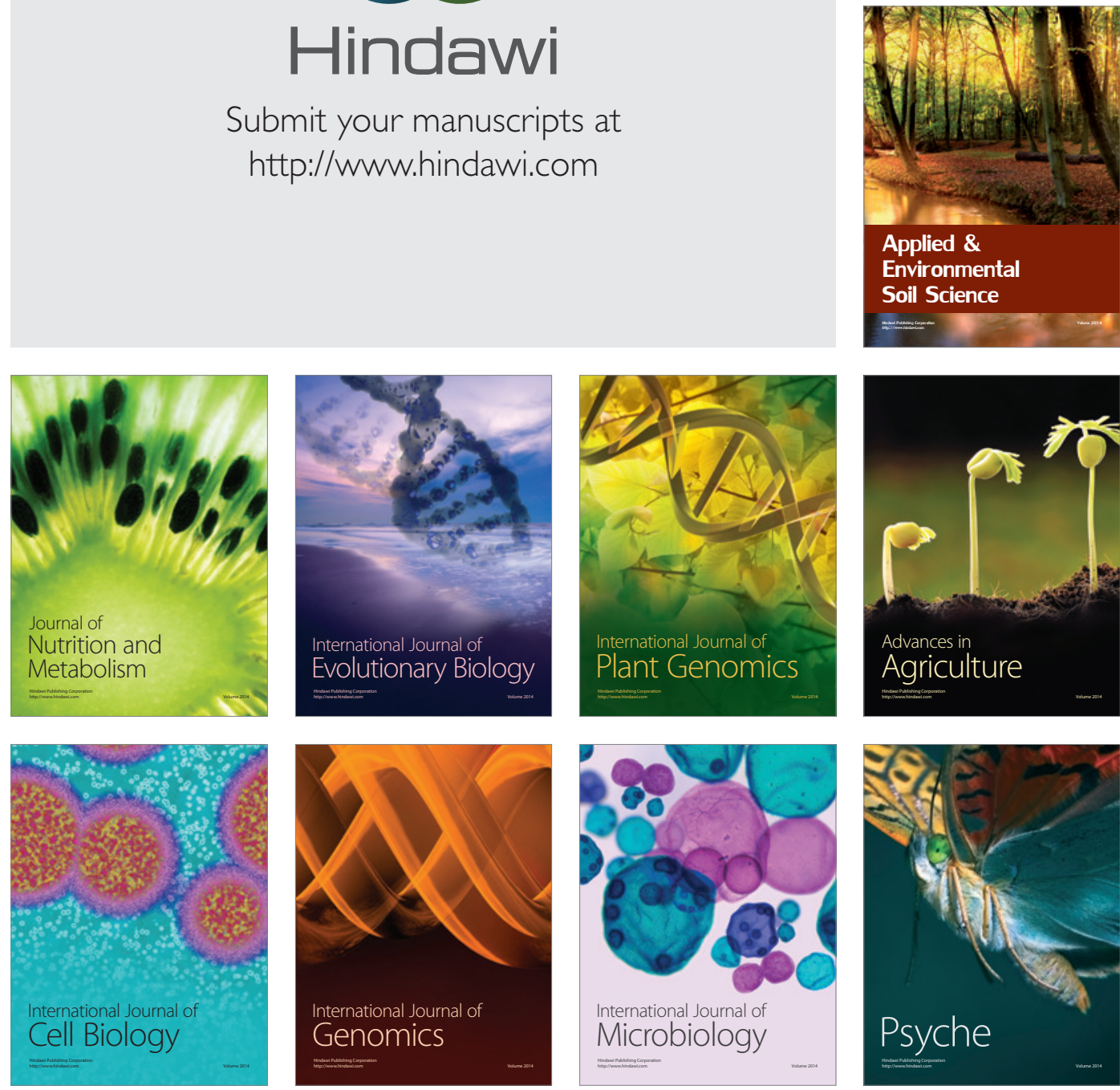
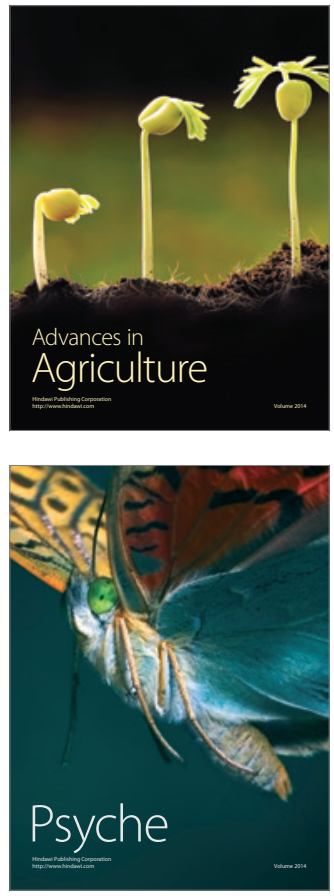